\title{
Augmentation of the anticancer activity of CYT997 in human prostate cancer by inhibiting Src activity
}

\author{
Yong Teng ${ }^{1,2,3^{*}}$, Yafei Cai ${ }^{4}$, Wenhu Pi ${ }^{3,5}$, Lixia Gao ${ }^{1}$ and Chloe Shay ${ }^{6}$
}

\begin{abstract}
Background: Abnormalities of tubulin polymerization and microtubule assembly are often seen in cancer, which make them very suitable targets for the development of therapeutic approach against rapidly dividing and aggressive cancer cells. CYT997 is a novel microtubule-disrupting agent with anticancer activity in multiple cancer types including prostate cancer. However, the molecular mechanisms of action of CYT997 in prostate cancer have not been well characterized.
\end{abstract}

Methods: Src knockdown cells were achieved by lentiviral-mediated interference. The drug effects on cell proliferation were measured by MTS. The drug effects on cell viability and death were determined by Cell Titer-Glo ${ }^{\oplus}$ Luminescent cell viability kit and flow cytometry with Zombie Aqua ${ }^{\mathrm{TM}}$ staining. The drug effects on apoptosis were assessed by Cell Death Detection Elisa kit and Western blot with a cleaved PARP antibody. The drug effects on cell invasion were examined by Matrigel-coated Boyden chambers. Oxidative stress was detected by DCFH-DA staining and electrochemical biosensor. Mouse models generated by subcutaneous or intracardiac injection were used to investigate the in vivo drug efficacy in tumor growth and metastasis.

Results: CYT997 effectively inhibited proliferation, survival, and invasion of prostate cancer cells via blocking multiple oncogenic signaling cascades but not the Src pathway. Inhibition of Src expression by small hairpin RNA or inactivation of Src by dasatinib increased the CYT997-induced cytotoxicity of in vitro. Moreover, the combination of dasatinib and CYT997 exhibited a superior inhibitory effect on tumor growth and metastasis compared with either of the drugs alone.

Conclusion: Our findings demonstrate that blockage of Src augments the anticancer effect of CYT997 on prostate cancer and suggest that co-treatment of dasatinib and CYT997 may represent an effective therapeutic regimen for limiting prostate cancer.

Keywords: CYT997, Prostate cancer, Dasatinib, Src, Anticancer, Synergistic treatment

\section{Background}

Prostate cancer, known as carcinoma of the prostate, is the most commonly diagnosed solid tumor among men. Despite new technology has enabled medical researchers to develop more reliable, less invasive screening and treatment methods, prostate cancer still remains the second leading cause of cancer-related death in male [1]. The failure of clinical treatment in patients with prostate

\footnotetext{
* Correspondence: yteng@augusta.edu

1Department of Oral Biology, Augusta University, Augusta, GA 30912, USA

${ }^{2}$ Georgia Cancer Center, Augusta University, 1120 15th Street, Augusta, GA 30912, USA

Full list of author information is available at the end of the article
}

cancer is often due to the heterogeneous nature of the disease. Comparative understanding of genetic pathways involved in the development and progression of prostate cancer may help define novel therapeutic targets or/and identify treatment regimens that are very likely to provide therapeutic benefit to patients.

Disrupting normal function of microtubules can engage the spindle checkpoint and arrest cell cycle progression at mitosis, leading to cell death [2]. Therefore, targeting microtubules is one of the efficient strategies for cancer treatment. A bunch of clinical chemo drugs, such as vinblastine, docetaxel, and paclitaxel, have 
potential to suppress microtubule dynamics without affecting microtubule polymer mass [2-4]. Although these microtubule-targeting agents (MTAs) are widely used to treat different types of human cancers [5-7], substantial drawbacks such as narrow therapeutic windows and lack of oral bioavailability still remain [8]. Moreover, the potential side effects (e.g., neural toxicity) and cardiovascular events (e.g., thromboembolism) largely limit therapeutic efficacy of MTA as single agents against cancer. To overcome these clinical problems, many research efforts have concentrated on developing novel MTAs. CYT997 is a new microtubule-disrupting agent screened from Cytopia's small molecule library, which has been proven to possess varying degrees of activity against cancers through inhibiting tubulin polymerization and disrupting cellular microtubules [9-11]. In phase I clinical trials, the safety, efficacy, and pharmacokinetics of CYT997 in cancer patients have been investigated [9].

Src, a non-receptor tyrosine kinase, has been implicated in a variety of different cancer types as well as in progression to malignancy [12-14]. Activation of Src is associated with its translocation to the plasma membrane in which Src interacts with a number of important effectors in response to extracellular signals. Regardless of which mechanism for Src activation, Src promotes numerous properties associated with metastatic potential once it is phosphorylated on tyrosine residues $[12,13]$. It has been reported that Src trafficking requires both microtubules and actin polymerization [15], which may link Src functions to MTAs. In hormonedependent cancers (e.g., breast and prostate cancer), steroid hormones trigger association of the androgen receptor (AR)-estradiol receptor (ER) complex with Src. A synthetic 10 amino-acid peptide that mimics the sequence of the SH3 domain-mediated binding of Src can prevent the AR/ER complex from associating with Src and inhibit the growth of LNCaP xenografts established in nude mice [16]. A study from Dr. Migliaccio's group shows that targeting the AR domain involved in AR/Src association impairs EGF signaling in human fibrosarcoma HT1080 cells [17]. Preclinical studies also established a role of Src in progressive stages of prostate cancer and cross talk between Src and AR signaling [18]. AR can also activate MAP kinase (MAPK) through an early and a late response pathway in a cell type-dependent manner [19]. The combination of the Src inhibitor PP2 and antiandrogen Casodex does not further decrease the invasion of the LNCaP derivative C4-2 cells [20], suggesting that $\mathrm{Src}$-assisted invasion may involve nongenomic AR actions whereby MAPK axis stimulation by AR signaling. Recent studies further reported that the Src inhibitors (e.g., dasatinib, saracatinib, and bosutinib) can inhibit motility, migration, and invasion of androgen-dependent and androgen-independent prostate cancer cells in a dose-dependent manner [21, 22].

In this study, we show that CYT997 effectively inhibits proliferation, viability, and invasion of prostate cancer cells. Genetic or pharmacological blockage of Src sensitizes prostate cancer cells towards CYT997 regardless of AR expression. Synergistic treatment with CYT997 and the Src inhibitor dasatinib exhibits a superior anticancer effect in mouse models of prostate cancer. These findings provide a molecular and cellular basis for CYT997 treatment, suggesting a clinical evaluation of CYT997 in combination with dasatinib for the treatment of patients with prostate cancer.

\section{Methods}

Cell lines, reagents, antibodies, and standard assays

Prostate cancer cell lines PC3, DU145, LNCaP, and 22Rv1 were obtained from ATCC and passage $<5$ were used in this study. LNCaP-derivative C4-2 and C4-2B cells were a gift from Dr. Daqing Wu (Georgia Cancer Center). All cells were maintained in Dulbecco's modified Eagle's medium (DMEM) containing 10\% fetal bovine serum (FBS). Luciferase stable PC3 cells were generated by transduction of pGL4.5 vector (Promoga, Madison, WI) encoding the luciferase reporter gene luc2 and selection of hygromycin. pLKO.1 lentiviral vectors harboring small hairpin RNAs (shRNAs) targeting Src were obtained from Open Biosystems (Huntsville, AL). CYT997 and dasatinib were purchased from Selleckchem (Houston, TX). 2',7'-Dichlorodihydrofluorescein diacetate (DCFH-DA) and D-Luciferin bioluminescent substrate were purchased from Sigma-Aldrich (St Louis, MO). Antibodies that recognize p62, p-AKT (Ser473), pERK1/2 (Thr202/Tyr204), p-STAT3 (Tyr705), p-Src (Tyr416), AKT, ERK1/2, STAT3, Src, LC3B I/II, and cleaved (c)-PARP were purchased from Cell Signaling Technology (Beverly, MA). $\beta$-Actin and Ki67 antibodies were purchased from Sigma-Aldrich (St Louis, MO) and Abcam (Cambridge, MA), respectively. Western blot, cell proliferation, and electrochemical detection were carried out as described previously [23-28]. Matrigel invasion assays were performed by transwells from $\mathrm{BD}$ biosciences (San Jose, CA) as described previously [2326]. Briefly, $5 \times 10^{4}$ serum-starved cells were seeded into Matrigel-coated Boyden chambers in the presence or absence of the indicated concentrations of CYT997, and DMSO containing 10\% FBS was added to the lower chamber. After 24-h treatment, the membranes that contained invading cells were fixed in methanol and stained with $0.2 \%$ Crystal violet. The dye was dissolved in $10 \%$ acetic acid and read colorimetrically at $590 \mathrm{~nm}$ for quantification of invasion. Cell viability was determined by CellTiter-Glo ${ }^{\circ}$ Luminescent cell viability assay (Promega, Madison, MI) and Zombie Aqua $^{\text {Tw }}$ fixable 
viability kit (BioLegend, San Diego, CA). Flow cytometry data were analyzed using FlowJo software (Tree Star, Ashland, OR).

\section{Detection of apoptosis}

The Cell Death Detection Elisa kit (Roche, Indianapolis, IN) was used to determine apoptosis by measuring mono- and oligonucleosomes in the lysates of apoptotic cells according to the manufacturer's protocol. Briefly, the cells treated with different drugs were lysed and placed into a streptavidin-coated microplate and incubated with a mixture of anti-histone-biotin and antiDNA-peroxidase. The amount of peroxidase retained in the immunocomplex was photometrically determined with 2,2'-azino-bis(3-ethylbenzothiazoline-6-sulphonic acid) (ABTS) as the substrate. Absorbance was measured at $405 \mathrm{~nm}$ (492 $\mathrm{nm}$ as reference wavelength).

\section{Animal models}

Six-week-old male NSG (NOD.Cg-Prkdc $c^{\text {scid }} I l 2 r g^{t m 1 W j l} /$ $S z J)$ mice were purchased from the Jackson Laboratory (Bar Harbor, ME), and all animal experiments were approved by the Institutional Animal Care and Use Committee (IACUC) of Augusta University. To generate a xenotransplantation model, exponentially growing PC3 cells $\left(1 \times 10^{6}\right.$ cells $)$ were suspended in $100 \mu \mathrm{l}$ PBS/matrigel (1:1) and injected subcutaneously into the right flanks of NSG mice. To generate an intracardiac model, NSG mice were injected via intracardiac with $1 \times 10^{6}$ luciferase-containing PC3 cells.

\section{Drug administration and immunohistochemistry}

Four days after PC3 cell implantation, mice were randomized to receive control vehicle and $\operatorname{drug}(\mathrm{s})(n=5)$. The treatment groups received followed equal volume treatment of CYT997 $(20 \mathrm{mg} / \mathrm{kg})$, dasatinib $(10 \mathrm{mg} / \mathrm{kg})$, or in combination of CYT997 $(20 \mathrm{mg} / \mathrm{kg})$ and dasatinib (10 mg/kg), respectively. CYT997 was administered by garage twice per day for a total of 3 weeks, and dasatinib was intraperitoneally (i.p.) administered 5 days per week for a total of 4 weeks. The control mice were injected i.p. with $100 \mu \mathrm{l}$ sterile saline. Tumor growth was measured externally every 4 to 7 days using vernier calipers as length $\times$ width $^{2} \times 0.52$. The mice were sacrificed on treatment day 42, and the lungs were removed and processed for immunohistochemistry (IHC) with Ki67 antibody and pathological analysis by $\mathrm{HE}$ staining as described previously [29, 30]. For intracardiac models, mice were randomized for vehicle (sterile saline) or combined (20 mg/kg CYT997 with $10 \mathrm{mg} / \mathrm{kg}$ dasatinib) treatment. Mice were imaged for luciferase signal by an intraperitoneal injection of luciferin $(15 \mu \mathrm{g}$ in $100 \mu \mathrm{l}$ PBS) every other week for 4 weeks using a Xenogen
IVIS-200 In Vivo Imaging System (PerkinElmer, Waltham, MA).

\section{Statistical analysis}

Treatment effects were evaluated using a two-tailed Student $t$ test at each measurement time point. To assess the longitudinal effect of treatment, a mixed model was employed to test the overall difference across all groups as well as between each pair of groups during the whole study period. The data were presented as means \pm SD from three or more independent experiments, and a $p$ value less than 0.05 was considered significant.

\section{Results}

CYT997 inhibits proliferation, viability, and invasion of prostate cancer cells through blocking multiple signaling pathways

CYT997 has been tested against a panel of 16 cancer cell lines and displays broad cytotoxicity in vitro [10]. To evaluate the drug effects on prostate cancer, prostate cancer cell lines with different genetic background were treated with various concentrations of CYT997. AR are highly expressed in 22Rv1, LNCaP, and its derivatives (C4-2 and C4-2B), and they are androgen-responsive cell lines. In contrast, DU145 and PC3 have relatively low basal levels of AR which are unresponsive to androgen stimulation. CYT997 inhibited proliferation and viability of all these cells in a dose-dependent manner (Fig. 1a, b and Additional file 1: Figure S1), suggesting that CYT997 exhibits inhibitory effects on cancer growth and survival regardless of AR expression. DU145 and PC3 are highly invasive prostate cancer cells, and their invasion potential was determined to explore the effect of CYT997 on cell motility. Transwell invasion assays showed that CYT997 effectively decreased cell invasion (Fig. 1c), suggesting CYT997 may block metastasis of prostate cancer. To determine the possible mechanisms involved in mediating drug action, we examined multiple oncogenic signaling pathways by Western blot. This investigation revealed a decreased phospho-activation of AKT and ERK1/2 following CYT997 treatment (Fig. 1d), which demonstrates that CYT997 can simultaneously suppress PI3K/AKT and MAPK pathways. Phosphorylation of STAT3 in PC3 cells remained undetectable regardless of CYT997 treatment; however, a sharp decrease in activated STAT3 was observed in DU145 cells when exposed to CYT997 (Fig. 1d).

\section{CYT997 induces apoptosis in prostate cancer cells}

Our pervious study has shown that sorafenib and other drugs approved for cancer treatment can cause mitochondrial dysfunction and increase intracellular oxidative stress [28]. We next examined whether CYT997 affects oxidative stress on prostate cancer cells. The 

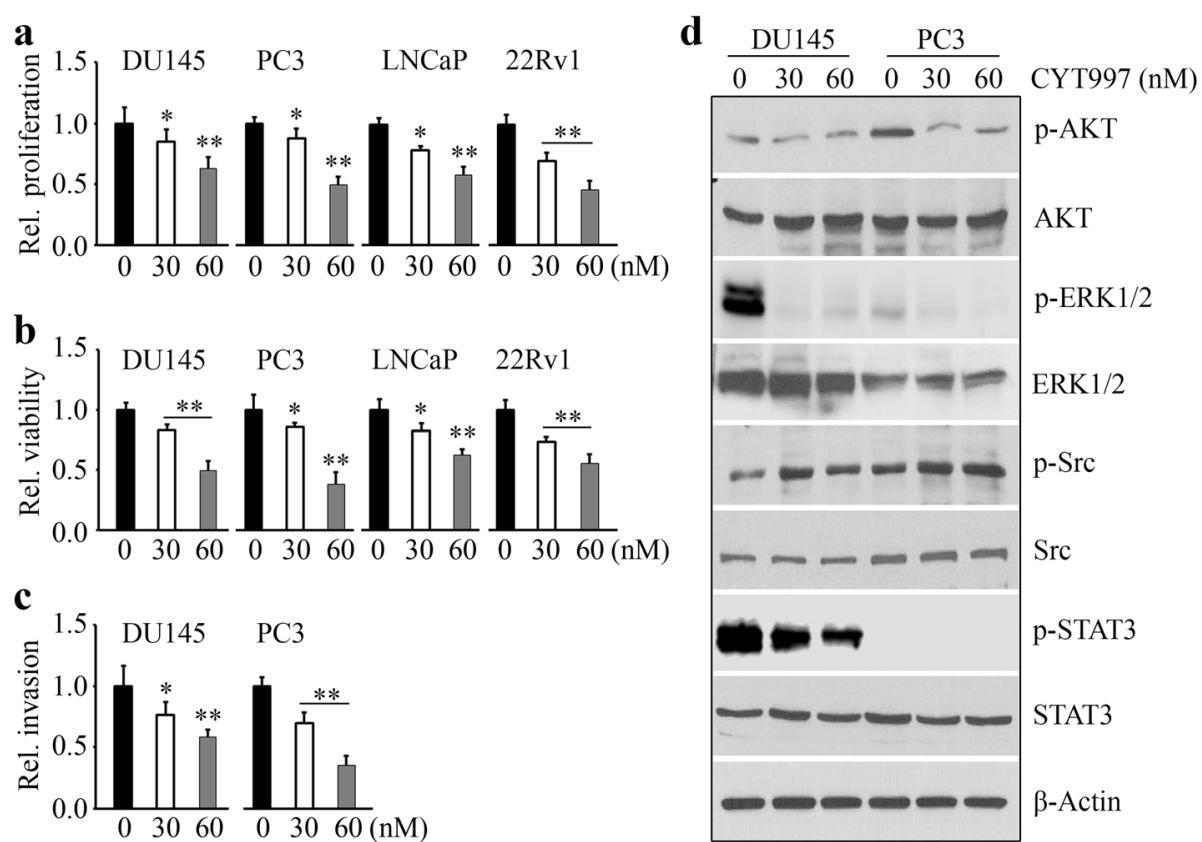

Fig. 1 CYT997 exhibits potent cytotoxicity against prostate cancer cells in vitro. a, b $1 \times 10^{4}$ prostate cancer cells (DU145, PC3, LNCaP, and 22Rv1) were seeded into 96-well plates and treated with the indicated concentrations of CYT997 for 48 h, and cell proliferation and viability were determined by MTS assays (a) and CellTiter-Glo Luminescent cell viability assays (b), respectively. c DU145 and PC3 cells were seeded into Matrigel-coated Boyden chambers in the presence or absence of the indicated concentrations of CYT997 for $24 \mathrm{~h}$, and invasion ability was quantitatively estimated by the absorbance value. d DU145 and PC3 cells were treated with CYT997 for 24 h, and cell lysates were collected for Western blot with the indicated antibodies

results from electrochemical biosensors showed that more superoxide $\left(\mathrm{O}_{2}^{-}\right)$were released in DU145 cells in the presence of CYT997 (Fig. 2a). In contrast, there was no difference in release of $\mathrm{O}_{2}^{-}$with or without CYT997 treatment (Fig. 2a). The cellular reactive oxygen species (ROS) evaluated by DCFH-DA further showed a significant increase of fluorescence in DU145 cells, but not in PC3 cells, following CYT997 exposure (Fig. 2b). These results are very consistent with the observations from electrochemical biosensors. To study whether CYT997 induces cell apoptosis, we determined the levels of cleaved PARP with or without CYT997 treatment. CYT997 led to a remarkable increase in cleaved PAPR in both DU145 and PC3 cells (Fig. 2c), which was confirmed with an increased apoptotic rate (Fig. 2d). Moreover, either DU145 or PC3 cells did not render autophagy in CYT997 treatment, as an evidence of no protein level changes in autophagic flux markers LC3B-II and p62 (Fig. 2c), suggesting that CYT997 induces apoptosis in prostate cancer cells via autophagy-independent mechanisms.

\section{Inhibition of Src augments the cytotoxicity of CYT997 in prostate cancer cells}

As shown in Fig. 1d and Additional file 2: Figure S2, phosphorylation levels of Src were increased in CYT997-treated DU145 and PC3 cells compared with non-treated control cells. These data prompted us to investigate whether inhibition of Src expression improves the CYT997 efficacy. We depleted Src in DU145 and PC3 cells using a shRNA strategy (Fig. 3a) and determined the consequences of Src loss in the presence of CYT997. Knockdown of Src not only inhibited cell proliferation and invasion but also enhanced CYT997induced suppression on these phenotypes (Fig. 3b, c), suggesting that blockage of Src may provide a clinical benefit for cancer patients following CYT997 treatment.

Dasatinib, an orally bioavailable synthetic small molecule inhibitor, can bind to and inhibit the growthpromoting activities of Src-family protein tyrosine kinases [21]. We thus co-treated prostate cancer cells with CYT997 and dasatinib. As would be expected, dasatinib attenuated CYT997-mediated phospho-activation of Src, with enhanced induction of cleaved PARP (Fig. 4a). Moreover, the combination of CYT997 and dasatinib acted more efficiently on cell death and apoptosis than either of the drugs alone (Fig. 4b, c). Figure 4d demonstrates that dasatinib augments the inhibitory effect of CYT997 on cell invasion.

\section{Dasatinib promotes the inhibitory effect of CYT997 on growth of prostate tumor xenograft in vivo}

Based on these encouraging data, we used mouse tumor models to evaluate the synergistic effects of CYT997 and 


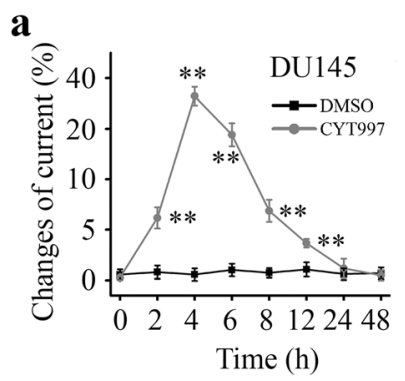

c

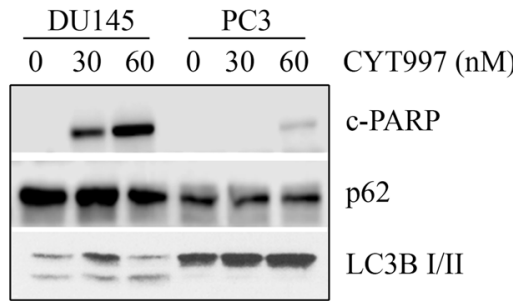

b
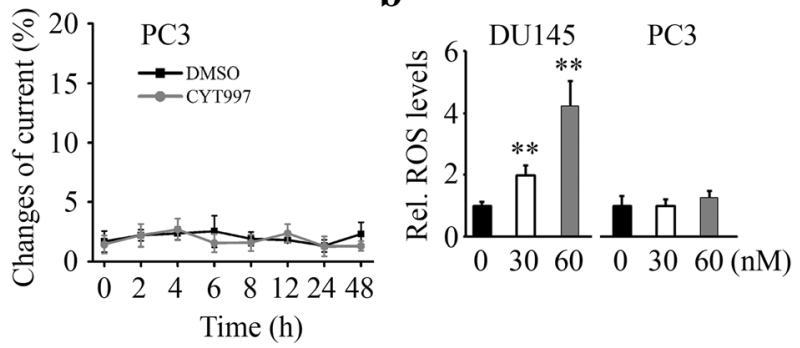

d

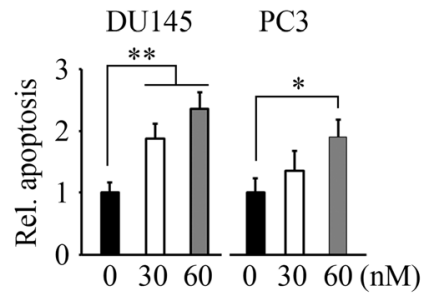

Fig. 2 CYT997 induces apoptosis but not autophagy in prostate cancer cells. a DU145 and PC3 cells were treated with 60 nM CYT997 for the indicated times, and $\mathrm{O}_{2}^{--}$release was determined by electrochemical biosensor. b DU145 and PC3 cells were treated with the indicated concentrations of CYT997 for $8 \mathrm{~h}$, and ROS generation was determined by DCFH-DA staining. $\mathbf{c}$, d DU145 and PC3 cells were treated with the indicated concentrations of CYT997 for 24 h, and cell lysates were collected for Western blot with the indicated antibodies (c), and apoptosis was determined by Cell Death Detection Elisa kit $(\mathbf{d}) .{ }^{*} p<0.05 ;{ }^{* *} p<0.01$

dasatinib on prostate cancer. The metastatic PC3 cells were implanted into the right flanks of NSG mice, and drug administration commenced on day 4 after implantation. Treatment with either CYT997 or dasatinib significantly reduced the size and weight of PC3-derived xenografts (Fig. 5a, b). The reduction in tumor growth was more significant in the mice receiving combined treatment compared with the mice receiving either drug alone (Fig. 5a, b). To determine whether Src signaling is involved in the synergistic a
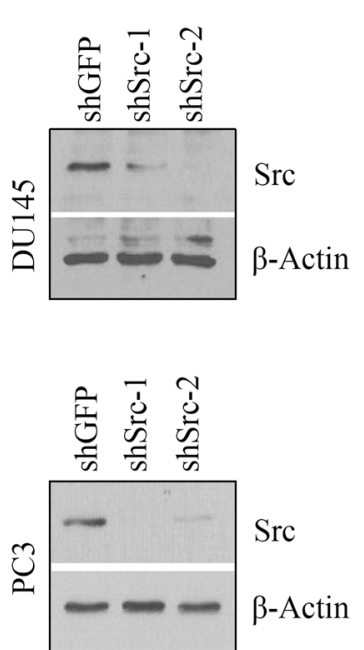

\section{b}

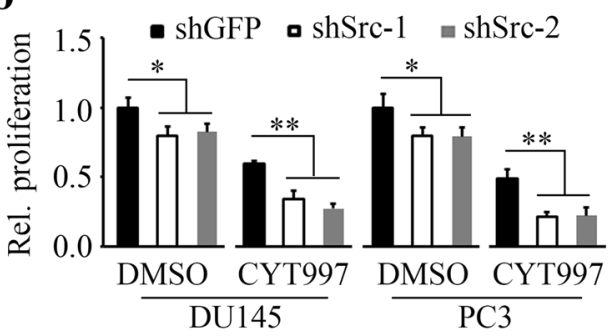

c

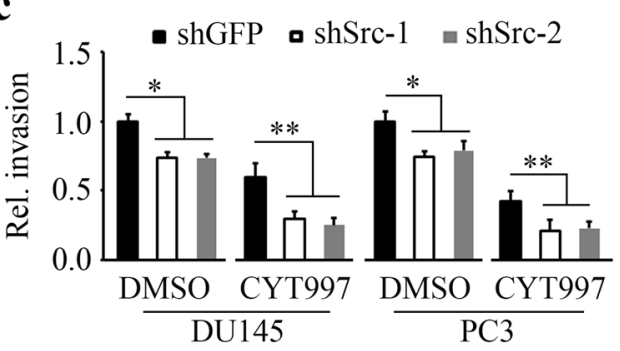

Fig. 3 Knockdown of Src sensitizes prostate cancer cells to CYT997. a The shRNAs against Src (shSrc-1, shSrc-2) were used to deplete Src expression in DU145 and PC3 cells. The shRNA against GFP (shGFP) was used as a negative control. After transfection and infection, the puromycin-resistant stable cells were verified for knockdown by Western blot. b, c Src knockdown and control cells were treated with $60 \mathrm{nM}$ CYT997. Cell proliferation was determined at $48 \mathrm{~h}$ after treatment by MTS assays (b), and cell invasion was determined at $24 \mathrm{~h}$ after treatment by Matrigel-coated modified Boyden chambers (c). ${ }^{*} p<0.05 ;{ }^{* *} p<0.01$ 

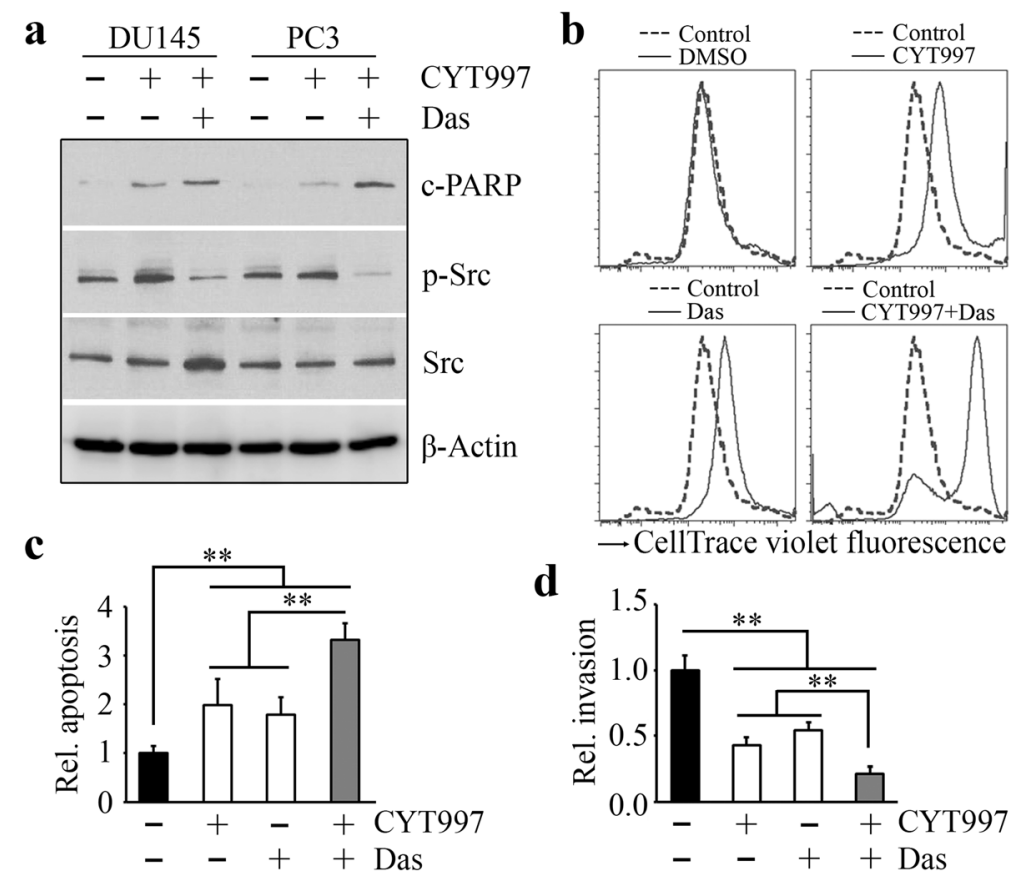

Fig. 4 Dasatinib enhances in vitro cytotoxicity of CYT997 in prostate cancer cells. a DU145 and PC3 cells were treated with 60 nM CYT997 for $24 \mathrm{~h}$, in the presence or absence of $50 \mathrm{nM}$ dasatinib (Das). Cell lysates were collected for Western blot with the indicated antibodies. b-d PC3 cells were treated with $60 \mathrm{nM}$ CYT997 for 24 h, in the presence or absence of $50 \mathrm{nM}$ dasatinib. Cell death was determined by flow cytometry with Zombie Aqua staining (b), apoptosis was determined by Cell Death Detection Elisa kit (c), and cell invasion was determined by Matrigelcoated modified Boyden chambers (d). ${ }^{* *} p<0.01$
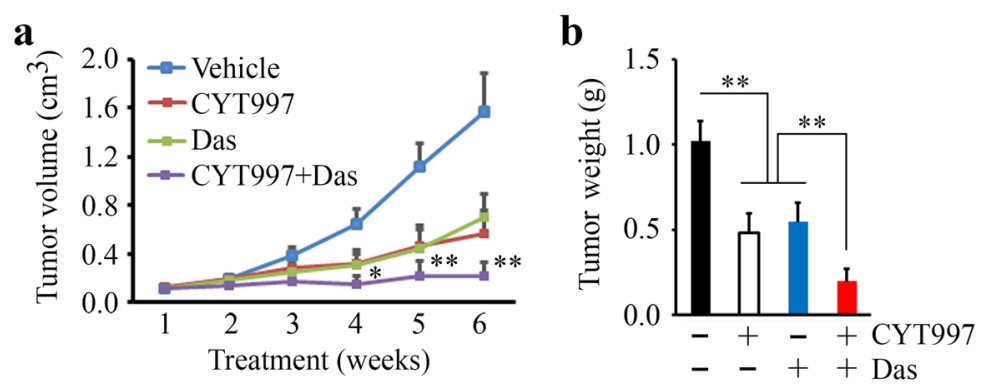

c

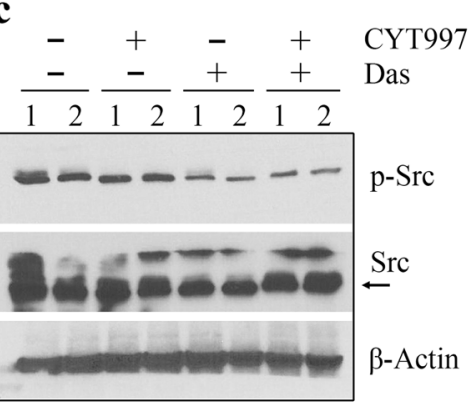

d
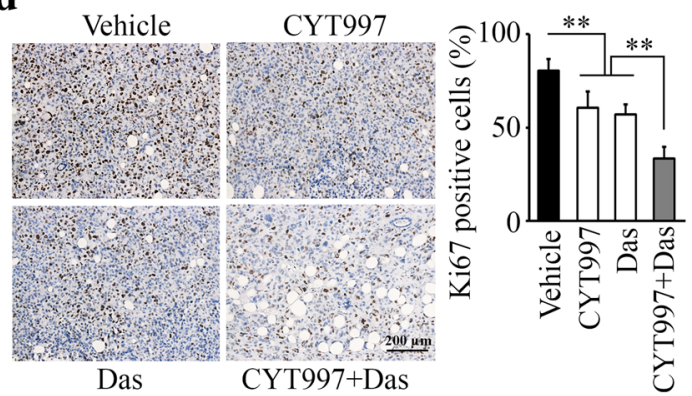

Fig. 5 Combination of CYT997 and dasatinib exhibits greater inhibitory activity of prostate tumor growth than either treatment alone. a, $\mathbf{b}$ When the PC3-derived flank xenografts have been established, NSG mice were randomly divided into four groups $(n=5)$ for the indicated treatments. Tumor growth was measured by tumor volume (a), and tumor burden at the end of the experiment was calculated as tumor weight (b). c, $\mathbf{d}$ Mice were sacrificed on day 42 after treatment, and xenografts were dissected and removed for Western blot with the indicated antibodies (c) and $\mathrm{IHC}$ with $\mathrm{Ki} 67$ antibody (d). In c, 1 and 2 indicate the tumor samples from two different mice. In d, representative images of $I H C$ are shown in the left panel, and quantitative data of staining intensity are shown in the right panel $(n=10) .{ }^{*} p<0.05 ;{ }^{* *} p<0.01$ 
drug effect, we examined Src activation using the cell lysates collected from the xenografts treated with CYT997 and dasatinib, alone or in combination. Western blot analysis showed that phosphorylation levels of Src were reduced in the dasatinib arm, but not in the CYT997 arm (Fig. 5c and Additional file 2: Figure S2). Dasatinib also blocked Src activation in CYT997 treatment (Fig. 5c and Additional file 2: Figure S2) and augmented CYT997induced repression of tumor growth. IHC analysis with a Ki67 antibody confirmed that this co-treatment has a superior inhibitory effect on prostate tumor growth compared to monotherapy (Fig. 5d).

\section{Combination of dasatinib and CYT997 strongly suppresses prostate tumor metastasis in vivo}

Our previous studies have demonstrated that tumor xenografts in NSG mice have potential to develop primary and metastatic tumors coincidently [29]. Therefore, we analyzed the mouse lungs and livers in xenograft model on day 42 after treatment. Incidence of metastatic nodules observed on the pulmonary and hepatic surface was significantly reduced in the mice treated with CYT997 or dasatinib compared with the mice treated with vehicle (Fig. 6a, c). The reduction in metastases in drug treatment was confirmed by histological analyses which showed decreased infiltrating tumors throughout the entire lung and liver (Fig. 6b, d). Combination of dasatinib and CYT997 displayed a stronger effect on suppression of metastasis than monotherapy, as evidenced by reduced nodule numbers on the pulmonary and hepatic surface (Fig. 6a, c) and relatively few, small tumor foci in the mice lung and liver (Fig. 6b, d). In order to detect the drug efficacy in bone metastasis, we generated a
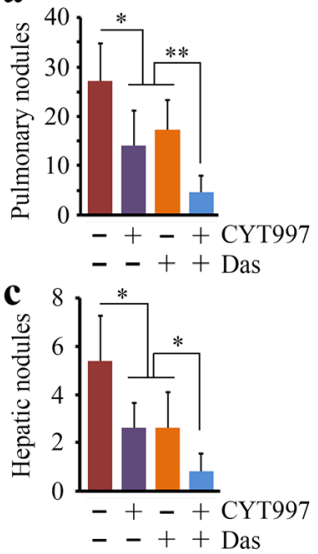

d
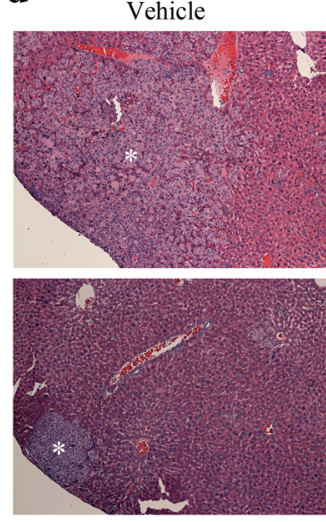

Das b

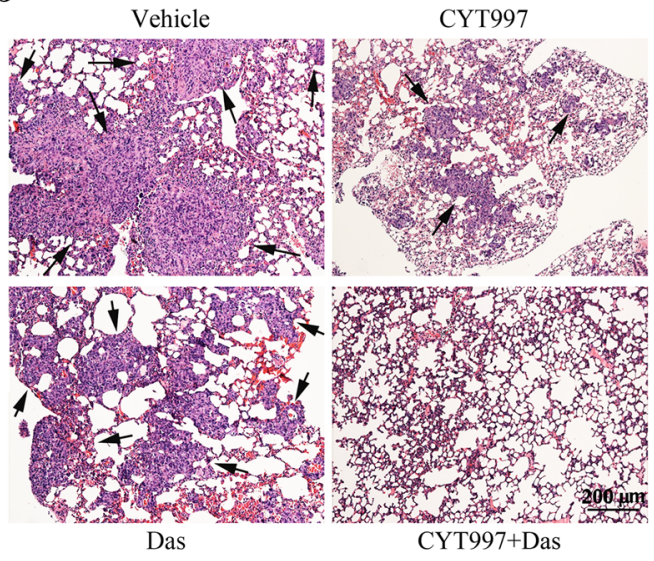

CYT997

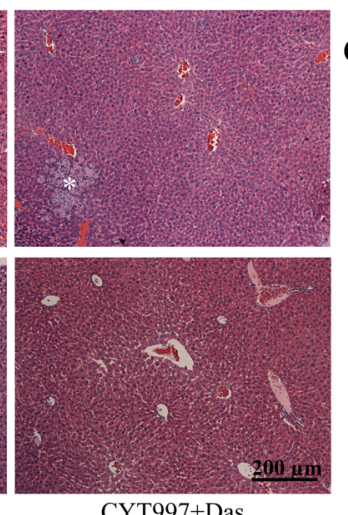

e

Vehicle CYT997+Das

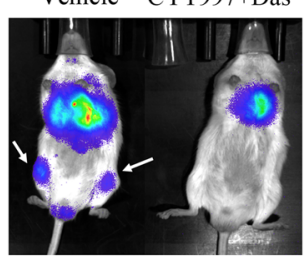

Fig. 6 Dasatinib improves CYT997 efficacy in suppression of prostate cancer metastasis. a-d The tumor-bearing mice described in Fig. 5 were sacrificed on day 42 after treatment, and the lungs and livers were dissected and removed. The metastatic nodules on the pulmonary or hepatic surface were counted $(\mathbf{a}, \mathbf{c})(n=5)$, and the tissue sections were stained with HE for pathological analysis (b, d). Black arrows in $\mathbf{b}$ indicate tumor foci in the mice lung, and white stars in $\mathbf{d}$ indicate tumor foci in the mice liver. e NSG mice were injected via intracardiac with $1 \times 10^{6}$ luciferase-containing PC3 cells before treatment. Tumor progression was monitored by examining luminescence in Xenogen IVIS-200 In Vivo Imaging System. ${ }^{*} p<0.05 ;{ }^{* *} p<0.01$ 
an experimental metastasis mouse model by intracardiac injection of luciferase-containing PC3 cells. This model allowed for determination of the process of metastatic colonization at the bone and other organ sites followed by bioluminescence imaging. Notably, PC3 cells established colonies in the bone compartment of the hind leg (Fig. 6e). In contrast to these observations, synergistic treatment almost completely blocked dissemination of PC3 cells to other organs (Fig. 6e). These data suggest that dasatinib would exert an additive anticancer effect with CYT997 in treatment of patients with prostate cancer.

\section{Discussion}

Microtubules are essential for cell growth, division, motility, intracellular trafficking, and the ability to adapt to a variety of shapes to interact with the environment [2]. Suppression of microtubule dynamics is a common mechanism of chemotherapeutic agents to block mitosis and kill tumor cells. CYT997, a novel anticancer MTA with a favorable combination of pharmacologic and pharmacokinetic properties and oral bioavailability, is currently undergoing clinical trials in a variety of cancer indications [9-11]. This work demonstrates the therapeutic efficacy of CYT997 in either cultured prostate cancer cells or mouse models of prostate cancer and reveals the possible mechanisms involved in drug action.

The therapeutic goal of cancer treatment has been to trigger tumor-selective cell death by apoptosis, and drug-mediated autophagy is increasingly recognized as an important factor in tumor apoptosis or survival [31]. Autophagic changes were not seen in the CYT997treated prostate cancer cells, excluding the possibility that CYT997 promotes apoptosis in prostate cancer cells by accumulation of autophagy. Oxidative stress is one of the other contributing factors to apoptosis. CYT997 induces an increase of ROS levels and $\mathrm{O}_{2}^{--}$release in DU145 cells, but not in PC3 cells. We also determined oxidative stress in AR-positive LNCaP cells with or without CYT997 treatment and observed the similar results seen in PC3 cells (data not shown). This discrepancy may be due to genetic background of the cell lines used in our study. One of our follow-up investigations is to discover possible molecular mechanisms mediating the apoptotic effect of CYT997.

SRC kinase activity has been found to be upregulated in a number of preclinical models of prostate cancer, and a potentially effective strategy to inhibit prostate cancer growth and metastasis is to target SRC kinases [21, 22]. Dasatinib inhibits multiple tyrosine kinases including SRC kinases, which are implemented to promote androgen independence in metastatic castration-resistant prostate cancer (mCRPC) that makes malignant cells unresponsive to therapies [32, 33]. Some of the common chemo drugs used to treat prostate cancer are MTAs. For example, docetaxel (Taxotere) stabilizes microtubules through binding to polymerized microtubules at the inner surface of the $\beta$ subunit $[34,35]$. As suggested by the phase III docetaxel plus dasatinib study, combination treatment is most effective in delaying and/or preventing skeletal metastases from prostate cancer [36]. However, no survival benefit was observed for the addition of dasatinib to docetaxel versus docetaxel/ placebo [36, 37]. Despite the disappointing results seen from studies evaluating dasatinib in combination with other agents, clinical trials are needed to address whether dasatinib may be used as a single agent or in combination with chemotherapy mediated by novel MTAs.

There is a clear potential for CYT997 to be used in all prostate tumors carrying AR or not and perhaps all clinical stages of prostate cancer since it can greatly inhibit nearly all prostate cancer cell lines. Our data indicate that CYT997 possess highly potent cytotoxic activity through inhibiting the PI3K/AKT and MAPK oncogenic signaling cascades. However, CYT997 cannot block the Src pathway at the examined concentrations. Considering that monotherapy frequently fails to produce an adequate response and combining cancer drugs with disparate mechanisms of action is a feasible strategy to achieve high anticancer activities, we treated prostate cancer cells with CYT997 and dasatinib. This drug combination shows synergistically decreased cell proliferation and increased apoptosis, leading to distinctly improved anticancer activities in vitro and in vivo. In the present study, we generated two types of prostate cancer mouse models using PC3 cells. The combination of CYT997 and dasatinib inhibits both prostate tumor growth and metastasis in these mouse models, providing a rational basis for the clinical treatment for patients with prostate cancer. Since the majority of mCRPC contains AR or even overexpresses it, use of different cells such as C4-2B (a cell line can produce osteoblastic metastases in the lumbar spine) would provide another model to explore the mechanisms of drug action and add value to the obtained findings. Future trials should identify patient subpopulations with elevated activation of Src in prostate cancer and better define the biologic processes of prostate cancer progression that are specifically "Src-driven." Combination treatment of CYT997 and dasatinib may be developed as a novel therapy to improve clinical outcomes for prostate cancer carrying high levels of activated Src.

\section{Conclusions}

Taking the obtained findings together, this work shows for the first time that blockage of Src by dasatinib can augment the anticancer efficacy of CYT997 in prostate cancer development and progression. Our study explicates a rational basis of synergistic actions of CYT997 and dasatinib and provides preclinical data for possible clinical application of this novel therapeutic strategy. In future work, we 
will improve drug delivery system to target prostate cancer more effectively and precisely.

\section{Additional files}

Additional file 1: Figure S1. CYT997 inhibits proliferation (a) and viability (b) of LNCaP derivative prostate cancer cell lines C4-2 and C4-2B. ${ }^{*} p<0.05 ;{ }^{* *} p<0.01$. (DOCX $132 \mathrm{~kb}$ )

Additional file 2: Figure S2. Quantitative data of phosphorylation Src levels in in vitro (a, representative images are shown in Fig. 1d) or in vivo treatment (b, representative images are shown in Fig. $5 c$ ). ${ }^{*} p<0.05 ;{ }^{* *} p<0.01$; $n=5$. (DOCX $127 \mathrm{~kb})$

\section{Abbreviations}

ABTS: 2,2'-Azino-bis(3-ethylbenzothiazoline-6-sulphonic acid); AR: Androgen receptor; DCFH-DA: 2',7'-Dichlorodihydrofluorescein diacetate; DMEM: Dulbecco's modified Eagle's medium; FBS: Fetal bovine serum; i.p.: Intraperitoneally; IHC: Immunohistochemistry; mCRPC: Metastatic castration-resistant prostate cancer; MTA: Microtubule-targeting agent; MTS: 3-(4,5-Dimethylthiazol-2-yl)-5-(3-carboxymethoxyphenyl)-2-(4-

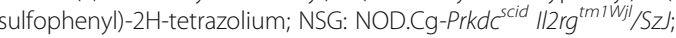
$\mathrm{O}_{2}^{-}$: Superoxide; ROS: Reactive oxygen species

\section{Acknowledgements}

We would like to thank Kongxiang Yang and Tian Jiang for the assistance with the data collection.

\section{Funding}

This work was supported in part by a grant from the Department of Defense (W81XWH-14-1-0412 to YT) and the National Natural Science Foundation of China (31372207 to YC)

\section{Availability of data and materials}

Not applicable.

\section{Authors' contributions}

$Y T, Y C, W P$, and LG performed the research and analyzed the results; CS discussed the results and edited the paper; YT designed the research, wrote the paper, and supervised the study. All authors read and approved the final manuscript.

\section{Competing interests}

The authors declare that they have no competing interests.

\section{Consent for publication}

Not applicable.

\section{Ethics approval and consent to participate}

All animal experiments were approved by the Institutional Animal Care and Use Committee (IACUC) of Augusta University.

\section{Publisher's Note}

Springer Nature remains neutral with regard to jurisdictional claims in published maps and institutional affiliations.

\section{Author details}

'Department of Oral Biology, Augusta University, Augusta, GA 30912, USA.

${ }^{2}$ Georgia Cancer Center, Augusta University, 1120 15th Street, Augusta, GA 30912, USA. ${ }^{3}$ Department of Biochemistry and Molecular Biology, Augusta University, Augusta, GA 30912, USA. ${ }^{4}$ College of Animal Science and Technology, Nanjing Agricultural University, Nanjing 210095, China. 5 Department of Radiation Oncology, Indiana University, Indianapolis, IN 46202, USA. ${ }^{6}$ Department of Pediatrics, Emory Children's Center, Emory University, Atlanta, GA 30322, USA.
Received: 27 February 2017 Accepted: 2 June 2017

Published online: 12 June 2017

\section{References}

1. Siegel RL, Miller KD, Jemal A. Cancer statistics, 2016. CA Cancer J Clin. 2016; 66(1):7-30.

2. Mukhtar $\mathrm{E}$, Adhami VM, Mukhtar $\mathrm{H}$. Targeting microtubules by natural agents for cancer therapy. Mol Cancer Ther. 2014;13(2):275-84.

3. Thomas E, Gopalakrishnan V, Hegde M, Kumar S, Karki SS, Raghavan SC, et al. A novel resveratrol based tubulin inhibitor induces mitotic arrest and activates apoptosis in cancer cells. Sci Rep. 2016:6:36345.

4. Dumontet $C$, Jordan MA. Microtubule-binding agents: a dynamic field of cancer therapeutics. Nat Rev Drug Discov. 2010;9(10):790-803.

5. Phillips GDL, Li G, Dugger DL, Crocker LM, Parsons KL, Mai E, et al. Targeting HER2-positive breast cancer with trastuzumab-DM1, an antibody-cytotoxic drug conjugate. Cancer Res. 2008;68(22):9280-90.

6. Sahra IB, Laurent K, Giuliano S, Larbret F, Ponzio G, Gounon P, et al. Targeting cancer cell metabolism: the combination of metformin and 2deoxyglucose induces p53-dependent apoptosis in prostate cancer cells. Cancer Res. 2010;70(6):2465-75.

7. Riechelmann H, Sauter A, Golze W, Hanft G, Schroen C, Hoermann K, et al. Phase I trial with the CD44v6-targeting immunoconjugate bivatuzumab mertansine in head and neck squamous cell carcinoma. Oral Oncol. 2008;44(9):823-9.

8. Lu Y, Chen J, Xiao M, Li W, Miller DD. An overview of tubulin inhibitors that interact with the colchicine binding site. Pharm Res. 2012;29(11):2943-71.

9. Burns CJ, Harte MF, Bu X, Fantino E, Joffe M, Sikanyika H, et al. Discovery of CYT997: a structurally novel orally active microtubule targeting agent. Bioorg Med Chem Lett. 2009;19(16):4639-42.

10. Burns CJ, Fantino E, Phillips ID, Su S, Harte MF, Bukczynska PE, et al. CYT997: a novel orally active tubulin polymerization inhibitor with potent cytotoxic and vascular disrupting activity in vitro and in vivo. Mol Cancer Ther. 2009; 8(11):3036-45.

11. Chen X, Yang C, Xu Y, Zhou H, Liu H, Qian W. The microtubule depolymerizing agent CYT997 effectively kills acute myeloid leukemia cells via activation of caspases and inhibition of PI3K Akt/mTOR pathway proteins. Exp Ther Med. 2013;6(2):299-304.

12. Yeatman TJ. A renaissance for SRC. Nat Rev Cancer. 2004:4(6):470-80.

13. Wheeler DL, lida M, Dunn EF. The role of Src in solid tumors. Oncologist. 2009:14(7):667-78

14. Gelman IH. Src-family tyrosine kinases as therapeutic targets in advanced cancer. Front Biosci. 2011:3:801-7.

15. Arnette C, Frye K, Kaverina I. Microtubule and actin interplay drive intracellular c-Src trafficking. PloS one. 2016;11(2):e0148996.

16. Migliaccio A, Varricchio L, De Falco A, Castoria G, Arra C, Yamaguchi H, et al. Inhibition of the $\mathrm{SH} 3$ domain-mediated binding of Src to the androgen receptor and its effect on tumor growth. Oncogene. 2007;26(46):6619-29.

17. Castoria G, Giovannelli P, Di Donato M, Hayashi R, Arra C, Appella E, et al. Targeting androgen receptor/Src complex impairs the aggressive phenotype of human fibrosarcoma cells. PloS one. 2013;8(10):e76899.

18. Varkaris A, Katsiampoura AD, Araujo JC, Gallick GE, Corn PG. Src signaling pathways in prostate cancer. Cancer Metastasis Rev. 2014;33(2-3):595-606.

19. Peterziel H, Mink S, Schonert A, Becker M, Klocker H, Cato AC. Rapid signalling by androgen receptor in prostate cancer cells. Oncogene. 1999;18(46):6322-9.

20. Asim M, Siddiqui I, Hafeez B, Baniahmad A, Mukhtar H. Src kinase potentiates androgen receptor transactivation function and invasion of androgen-independent prostate cancer C4-2 cells. Oncogene. 2008:27(25): 3596-604.

21. Nam S, Kim D, Cheng JQ, Zhang S, Lee JH, Buettner R, et al. Action of the Src family kinase inhibitor, dasatinib (BMS-354825), on human prostate cancer cells. Cancer Res. 2005;65(20):9185-9.

22. Rabbani SA, Valentino ML, Arakelian A, Ali S, Boschelli F. SKI-606 (Bosutinib) blocks prostate cancer invasion, growth, and metastasis in vitro and in vivo through regulation of genes involved in cancer growth and skeletal metastasis. Mol Cancer Ther. 2010;9(5):1147-57.

23. Xie X, Tang SC, Cai Y, Pi W, Deng L, Wu G, et al. Suppression of breast cancer metastasis through the inactivation of ADP-ribosylation factor. Oncotarget. 2016:7:14-6.

24. Teng Y, Mei Y, Hawthorn L, Cowell JK. WASF3 regulates miR-200 inactivation by ZEB1 through suppression of KISS1 leading to increased invasiveness in breast cancer cells. Oncogene. 2014;33(2):203-11. 
25. Teng Y, Pi W, Wang Y, Cowell JK. WASF3 provides the conduit to facilitate invasion and metastasis in breast cancer cells through HER2/HER3 signaling. Oncogene. 2016;35(35):4633-40.

26. Shi L, Zhang W, Zou F, Mei L, Wu G, Teng Y. KLHL21, a novel gene that contributes to the progression of hepatocellular carcinoma. BMC Cancer. 2016;16(1):815.

27. Teng Y, Qin H, Bahassan A, Bendzunas NG, Kennedy EJ, Cowell JK. The WASF3-NCKAP1-CYFIP1 complex is essential for breast cancer metastasis. Cancer Res. 2016;76(17):5133-42.

28. Gao L, Wang $X$, Tang Y, Huang S, Hu CAA, Teng Y. FGF19/FGFR4 signaling contributes to the resistance of hepatocellular carcinoma to sorafenib. J Exp Clin Cancer Res. 2017;36(1):8.

29. Teng Y, Ren X, Li H, Shull A, Kim J, Cowell JK. Mitochondrial ATAD3A combines with GRP78 to regulate the WASF3 metastasis-promoting protein. Oncogene. 2016;35(3):333-43.

30. Davis JE, Xie X, Guo J, Huang W, Chu WM, Huang S, et al. ARF1 promotes prostate tumorigenesis via targeting oncogenic MAPK signaling. Oncotarget. 2016;7(26):39834-45.

31. Gao L, Jauregui $C E$, Teng Y. Targeting autophagy as a strategy for drug discovery and therapeutic modulation. Future Med Chem. 2017;9(3):335-45.

32. Twardowski PW, Beumer JH, Chen C, Kraft AS, Chatta GS, Mitsuhashi M, et al. A phase II trial of dasatinib in patients with metastatic castration-resistant prostate cancer treated previously with chemotherapy. Anti-Cancer Drug. 2013;24(7):743-53

33. Araujo JC, Trudel GC, Paliwal P. Long-term use of dasatinib in patients with metastatic castration-resistant prostate cancer after receiving the combination of dasatinib and docetaxel. Cancer Manag Res. 2013;6:25-30.

34. Tannock IF, de Wit R, Berry WR, Horti J, Pluzanska A, Chi KN, et al. Docetaxel plus prednisone or mitoxantrone plus prednisone for advanced prostate cancer. N Engl J Med. 2004;351(15):1502-12.

35. James ND, Sydes MR, Clarke NW, Mason MD, Dearnaley DP, Spears MR, et al. Addition of docetaxel, zoledronic acid, or both to first-line long-term hormone therapy in prostate cancer (STAMPEDE): survival results from an adaptive, multiarm, multistage, platform randomised controlled trial. Lancet. 2016;387(10024):1163-77.

36. Araujo JC, Trudel GC, Saad F, Armstrong AJ, Evan YY, Bellmunt J, et al. Docetaxel and dasatinib or placebo in men with metastatic castrationresistant prostate cancer (READY): a randomised, double-blind phase 3 trial. The Lancet Oncol. 2013:14(13):1307-16.

37. Agarwal N, Di Lorenzo G, Sonpavde G, Bellmunt J. New agents for prostate cancer. Ann Oncol. 2014;25(9):1700-9.

\section{Submit your next manuscript to BioMed Central and we will help you at every step:}

- We accept pre-submission inquiries

- Our selector tool helps you to find the most relevant journal

- We provide round the clock customer support

- Convenient online submission

- Thorough peer review

- Inclusion in PubMed and all major indexing services

- Maximum visibility for your research

Submit your manuscript at www biomedcentral.com/submit

) Biomed Central 